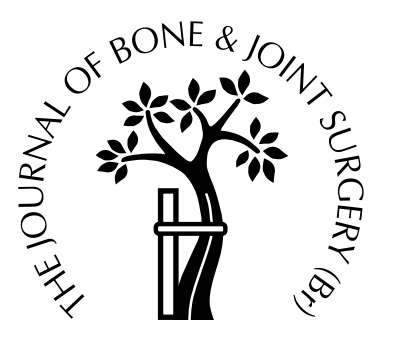

\title{
Pyomyositis of the iliacus muscle in a child
}

\author{
W. R. C. Peckett, A. Butler-Manuel, L. A. Apthorp \\ From Conquest Hospital, St Leonards-on-Sea, England
}

$\mathbf{P}$ yomyositis is rarely seen in temperate climates. Typically, it presents with the formation of an abscess requiring surgical drainage and it has been reported as a differential diagnosis for septic arthritis of the hip.

We describe the occurrence of pyomyositis of the iliacus muscle in a ten-year-old girl which was diagnosed by MRI and blood culture. Formation of an abscess did not occur despite marked focal inflammation and swelling of the muscle. Conservative treatment with antibiotics alone led to complete clinical and radiological resolution of the infection.

We could find no previous description of pyomyositis in a child in the British orthopaedic literature. Orthopaedic surgeons, particularly those with a paediatric interest, should be aware of this condition and its presentation, diagnosis and treatment.

J Bone Joint Surg [Br] 2000;82-B:103-5.

Received 24 February 2000; Accepted after revision 4 May 2000

Pyomyositis (tropical pyomyositis) is a primary bacterial infection of skeletal muscle which is not secondary to a contiguous infection of the skin, bone or soft tissue. ${ }^{1}$ It usually affects the muscles around the hip and thigh, ${ }^{2}$ and initially may be mistakenly diagnosed as septic arthritis of the hip. ${ }^{1,3-5}$ Adults between the ages of 20 and 40 years are most commonly affected. ${ }^{3}$ It has been described in children in tropical and subtropical climates but only rarely in temperate climates. ${ }^{1,3-7}$

We describe the occurrence of a case of pyomyositis of the iliacus muscle in a child in the UK. To our knowledge this has not previously been reported in the British orthopaedic literature.

W. R. C. Peckett, FRCS, Specialist Registrar

A. Butler-Manuel, FRCS, Consultant Orthopaedic Surgeon

L. A. Apthorp, FRCR, Consultant Radiologist

Conquest Hospital, The Ridge, St Leonards-on-Sea, East Sussex TN37 7RD, UK.

Correspondence should be sent to Mr W. R. C. Peckett.

C2001 British Editorial Society of Bone and Joint Surgery 0301-620X/00/111095\$2.00

\section{Case report}

A ten-year-old Caucasian girl was referred by her general practitioner with a two-week history of increasing pain in the left side of her lower back radiating to the left buttock, the posterior area of the thigh and the knee. The pain had begun after a vigorous physical exercise (PE) lesson at school and had gradually increased until she was unable to bear weight. She was admitted to hospital.

There was no history of recent foreign travel, prodromal illness or any other significant medical or family history.

On examination she was distressed, in pain and afebrile. The left lower back and the sacroiliac and buttock areas were tender. The range of movement of the left hip was markedly reduced, particularly flexion, although rotation was free in extension. On the right the straight-leg raise was to $90^{\circ}$ at which point pain was felt in the left lower back. That on the left was limited to $10^{\circ}$ because of severe pain in the left lower back and buttock. Neurological examination was normal.

Initially, haematological investigations showed a leucocytosis of $16.6 \pm 10^{3}$ per $\mathrm{mm}^{3}$, a neutrophil count of 13.50 $\pm 10^{3}$ per $\mathrm{mm}^{3}$ (normal 2.0 to $7.5 \times 10^{3}$ per $\mathrm{mm}^{3}$ ), an ESR of $51 \mathrm{~mm} / \mathrm{hr}$ and a C-reactive protein level of $90 \mathrm{mg} / \mathrm{l}$ (normal $<10 \mathrm{mg} / \mathrm{l}$ ). Urine microscopy and culture proved negative.

Overnight, she had a pyrexia of $38.5^{\circ} \mathrm{C}$ and the following day her condition remained unchanged. We carried out blood cultures and screening tests for anti-streptolysin $\mathrm{O}$ and rheumatoid factor. A provisional diagnosis of acute infective lumbar discitis or acute sacroiliitis was made.

An MR scan was carried out. This showed an enlarged left iliacus muscle with a low signal on the T1-weighted and a high signal on the T2-weighted images (Fig. 1). The findings were thought to be consistent with localised inflammation of the muscle, in the absence of an abscess or any other abnormality in the surrounding bones and joints including the left hip and sacroiliac joint. The diagnosis of pyomyositis was confirmed when Staphyloccus aureus was isolated from the blood cultures.

She was initially treated by bed rest and with intravenous Magnapen, $500 \mathrm{mg}$ every six hours, which was changed to flucloxacillin $500 \mathrm{mg}$ intravenously when Staphyloccus aureus was isolated. By the fifth day after admission her 


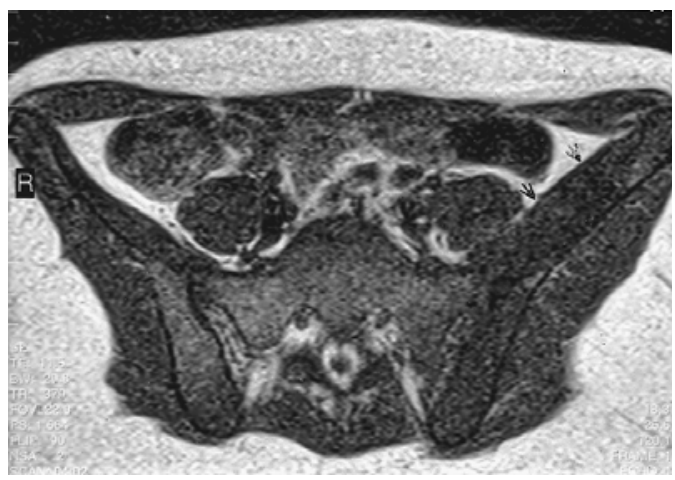

Fig. 1a

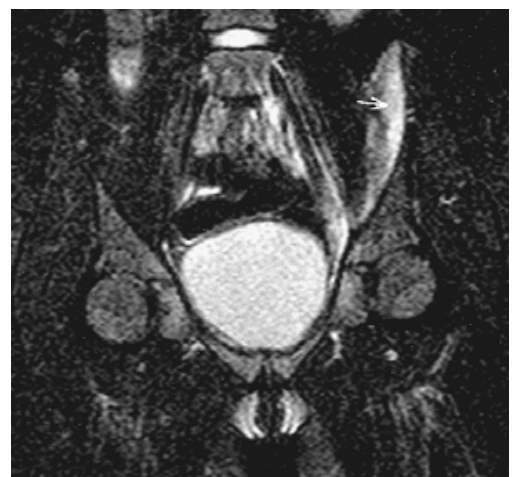

Fig. 1b

MRI showing a) the axial T1-weighted image with an enlarged, relatively low signal in the left iliacus muscle (arrows) and b) the coronal FATSAT T2-weighted image with a high signal within the enlarged left iliacus muscle indicating muscle oedema (arrow).

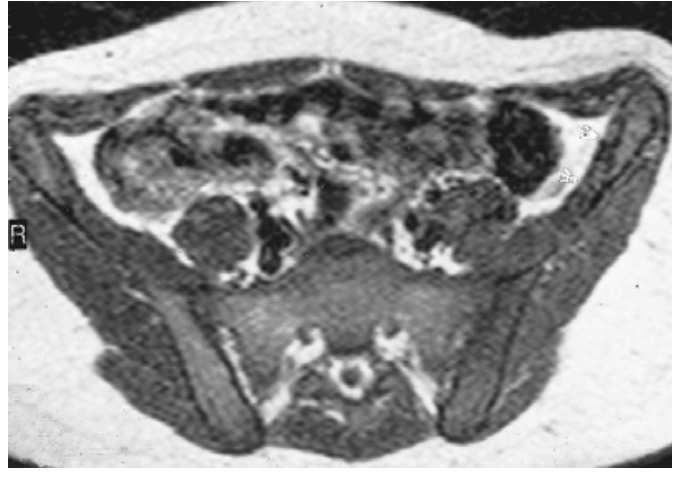

Fig. 2a

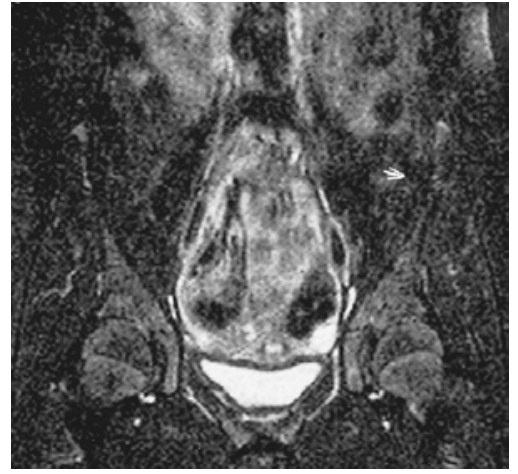

Fig. 2b

MRI showing a) the axial T1-weighted and b) the coronal FATSAT images with resolution of the previously identified changes and a normal left iliacus muscle (arrows).

pain had markedly improved; she was able to walk independently partially weight-bearing on crutches, and had been apyrexial for 48 hours. At this stage she was discharged home on oral flucloxacillin. One week later her pain had almost completely resolved and at six weeks she was asymptomatic. Her full blood count, ESR, CRP and MRI at six weeks were all normal (Fig. 2).

\section{Discussion}

Pyomyositis is a condition usually seen in hot climates and commonly affects the muscles around the hip. ${ }^{1,3,4,6-9}$ It has rarely been reported in adults in the UK. ${ }^{4,10,11}$ A review of the literature revealed only one case previously described in the UK, in a nine-year-old boy who presented with an abscess in the psoas muscle which was diagnosed by CT and required surgical drainage. ${ }^{12}$

MRI was crucial in making an early diagnosis. The high signal on the T2-weighted and the low signal on the T1weighted images confirmed the florid inflammation within the iliacus muscle, without formation of an abscess. It has been recognised as the radiological investigation of choice $^{13}$ in the diagnosis of pyomyositis. In addition to demonstrating the abnormal iliacus muscle, it was possible to rule out associated infection in the pelvis and hips.

Our findings contrast with those of other reports in which presentation has often been with an abscess requiring surgical drainage. ${ }^{1-5,8,9,14,15}$ Most of these cases did not have the benefit of MRI. Predisposing factors were often present such as skin conditions, ${ }^{2}$ pyarthrosis, ${ }^{2}$ recent surgery and wound dehiscence, ${ }^{2}$ diabetes, ${ }^{2,4,9}$ childbirth and epidural cannulation, $^{13}$ and HIV disease. ${ }^{11}$ There were no such features in our case, which appeared to be a primary infection of muscle with no secondary spread from adjacent structures.

Pyomyositis affecting the iliacus muscle and the adductor muscles of the hip has been described as a differential diagnosis for septic arthritis of the hip. ${ }^{1,3-5}$ Our patient presented with left-sided low back, buttock and thigh pain and the free movement of the left hip in extension made septic arthritis unlikely. The iliacus muscle is closely associated with the psoas muscle, the femoral and lateral femoral cutaneous nerves, ${ }^{14}$ the hip and pelvic and intra-abdominal structures. Pathological abnormality in the iliacus muscle may present, as in our patient, with lower abdominal pain, pain in the hip or femoral neuropathy. 
Staphylococcus aureus was the infecting organism and is responsible for pyomyositis in approximately $90 \%$ of cases. $^{3,8}$ It is not clear why there was no progression to formation of an abscess since the condition had been developing over two weeks. This is comparable to the range of three days to three weeks which has been described elsewhere and in which the primary presentation was usually with an abscess. ${ }^{3}$ It may be that primary pyomyositis occurring in a deeply-sited muscle is a different clinical entity from the formation of an abscess in more superficial muscles, which are more susceptible to the spread of infection from adjacent skin lesions or puncture wounds.

The history of previous physical exercise may or may not be relevant. It is possible that a 'minor' strain of the iliacus in some way made the muscle more susceptible to transient bacteraemia. This association has been recognised previously. ${ }^{2}$ The initial MR scan showing a low signal on the T1-weighted and a high signal on the T2-weighted images in the iliacus is consistent with inflammation and not haemorrhage.

Pyomyositis has been reported in children in temperate climates, and in a British child in the paediatric literature. ${ }^{12}$ It should be considered in the differential diagnosis of any child with pyrexia complaining of joint pain or muscle aches, particularly as a differential diagnosis in septic arthritis of the hip. Orthopaedic surgeons should be aware of this condition. MRI is invaluable in establishing the diagnosis and its anatomical site and in helping to exclude other possible diagnoses.

No benefits in any form have been received or will be received from a commercial party related directly or indirectly to the subject of this article.

\section{References}

1. Chen W-S, Wan Y-L. Iliacus pyomyositis mimicking septic arthritis of the hip joint. Arch Orthop Trauma Surg 1996;115:233-5.

2. Hall RL, Callaghan JJ, Moloney E, Martinez S, Harrelson JM. Pyomyositis in a temperate climate: presentation, diagnosis and treatment. J Bone Joint Surg [Am] 1990;72-A:1240-4.

3. Renwick SE, Ritterbusch JF. Pyomyositis in children. J Pediatr Orthop 1993;13:769-72.

4. Andrew JG, Czyz WM. Pyomyositis presenting as septic arthritis: a report of 2 cases. Acta Orthop Scand 1998;59:587-8.

5. De Boeck H, Noppen L, Desprechins B. Pyomyositis of the adductor muscles mimicking an infection of the hip: diagnosis by magnetic resonance imaging: a case report. J Bone Joint Surg [Am] 1994;76-A:747-50.

6. Chacha PB. Muscle abscesses in children. Clin Orthop 1970;70: 174-80.

7. Chiedozi LC. Pyomyositis: review of 205 cases in 112 patients. Am J Surg 1979;137:255-9.

8. Birkbeck D, Watson JT. Obturator internus pyomyositis: a case report. Clin Orthop 1995;316:221-6.

9. Freedman KB, Hahn GV, Fitzgerald RH. Unusual case of septic arthritis of the hip: spread from adjacent adductor pyomyositis. $J$ Arthroplasty 1999;14:886-91.

10. Rogers DW. Case of pyomyositis occurring in London. Br Med $\mathrm{J}$ 1973;3:679.

11. Watts RA, Hoffbrand BI, Paton DF, Davis JC. Pyomyositis associated with human immunodeficiency virus infection. $\mathrm{Br}$ Med $J$ 1987;294:1524-5.

12. O'Reilly M, Cook JV, O'Reilly PM, Wood BP. Radiological case of the month: psoas pyomyositis. Arch Pediatr Adolesc Med 1996;150:1305-6.

13. Yuh WTC, Schreiber AE, Montgomery WJ, Ehara S. Magnetic resonance imaging of pyomyositis. Skeletal Radiol 1988;17:190-3.

14. Pai VS, Yee E, Lawson D, Curtis G. Iliacus pyomyositis with involvement of lateral cutaneous nerve of the thigh. $N \mathrm{Z} \mathrm{Med} J$ 1994;107:378-9.

15. Kinahan AM, Douglas JM. Piriformis pyomyositis mimicking epidural abscess in a parturient. Can J Anaesth 1995;42:240-5. 